\title{
TITLE:
}

\section{Free-energy analysis of solubilization in micelle}

$\operatorname{AUTHOR}(S)$ :

Matubayasi, N; Liang, KK; Nakahara, M

\section{CITATION:}

Matubayasi, N ...[et al]. Free-energy analysis of solubilization in micelle. JOURNAL OF CHEMICAL PHYSICS 2006, 124(15): 154908.

\section{ISSUE DATE:}

2006-04-21

URL:

http://hdl.handle.net/2433/50356

\section{RIGHT:}

Copyright 2006 American Institute of Physics. This article may be downloaded for personal use only. Any other use requires prior permission of the author and the American Institute of Physics. 


\title{
Free-energy analysis of solubilization in micelle
}

\author{
Nobuyuki Matubayasi, ${ }^{a}$ Kuo Kan Liang, and Masaru Nakahara \\ Institute for Chemical Research, Kyoto University, Uji, Kyoto 611-0011, Japan
}

(Received 29 December 2005; accepted 20 February 2006; published online 20 April 2006)

\begin{abstract}
A statistical-mechanical treatment of the solubilization in micelle is presented in combination with molecular simulation. The micellar solution is viewed as an inhomogeneous and partially finite, mixed solvent system, and the method of energy representation is employed to evaluate the free-energy change for insertion of a solute into the micelle inside with a realistic set of potential functions. Methane, benzene, and ethylbenzene are adopted as model hydrophobic solutes to analyze the solubilization in sodium dodecyl sulfate micelle. It is shown that these solutes are more favorably located within the micelle than in bulk water and that the affinity to the micelle inside is stronger for benzene and ethylbenzene than for methane. The micellar system is then divided into the hydrophobic core, the head-group region in contact with water, and the aqueous region outside the micelle to assess the relative importance of each region in the solubilization. In support of the pseudophase model, the aqueous region is found to be unimportant to determine the extent of solubilization. The contribution from the hydrophobic-core region is shown to be dominant for benzene and ethylbenzene, while an appreciable contribution from the head-group region is observed for methane. The methodology presented is not restricted to the binding of a molecule to micelle, and will be useful in treating the binding to such nanoscale structures as protein and membrane. (C) 2006 American Institute of Physics. [DOI: 10.1063/1.2186324]
\end{abstract}

\section{INTRODUCTION}

Micelle is a self-assembled aggregate of amphiphilic surfactants in water. It involves a hydrophobic core and provides a favorable environment for organic compounds. Upon formation of a micelle, the solubility is often enhanced for an organic compound which is insoluble or sparingly soluble in water. This phenomenon is called solubilization, and is a most important function of a micelle. ${ }^{1-3}$ The solubilization results from the contrast of the interactions among water, surfactants, and organic compounds. A well-defined statistical-mechanical framework is thus desirable to be developed for the molecular understanding and control of the solubilization.

Since the solubilization is of central importance in interface sciences and technologies, it has been extensively studied in strong connections to pharmaceutical and biological applications. ${ }^{1-3}$ In the past, however, an enormous set of experimental data on the solubilization were processed with phenomenological or coarse-grained models. ${ }^{1-6}$ A statisticalmechanical framework could not be implemented in practice when a direct reference to intermolecular interactions was desired. In the present, a theoretical-computational treatment of a micellar solution is feasible at atomic resolution with the advance of molecular simulation methodology. ${ }^{7-25}$ The structure and dynamics of a micellar aggregate and the surrounding medium can be quantitatively analyzed through a nanoscale simulation. In this paper, we provide a molecular approach to the solubilization. We extend the method of energy representation developed in previous papers, ${ }^{26-28}$ and com-

\footnotetext{
a) Author to whom correspondence should be addressed. Electronic mail: nobuyuki@scl.kyoto-u.ac.jp
}

bine it with a large-scale molecular simulation to evaluate the solubility change induced by micelle formation.

The basic idea of our scheme is to view a micellar solution as a mixed solvent. The surfactant molecules are treated not as solute species, but as part of the mixed solvent system. In this view, the solvation free energy of a solute molecule of interest in a micellar solution denotes the free-energy change for turning on the interactions of the solute with the solvent water, surfactants, and counterions if present. The solubilization is described by the difference in the solvation free energy between the micellar system and the neat solvent (pure water) system. When the micelle is dilute, in particular, it is natural to set the origin of the solution at (or near) the micellar center. The mixed solvent system then consists of the surfactant molecules forming the micelle, water, and distributed counterions (when the surfactant is ionic). Water is deficient in the interior of the micelle, and the surfactant molecules belonging to the micelle are localized around the origin and are finite in number. The mixed solvent is thus inhomogeneous even before the solute insertion, and is partially finite in the sense that the number is finite for the surfactant (and counterion) involved as solvent species in the dilute micellar solution. The point of our scheme is to evaluate the solvation free energy of a solute in an inhomogeneous and partially finite, mixed solvent system.

Even when the system is inhomogeneous and partially finite, a formally exact free energy calculation is possible by the standard free-energy perturbation and thermodynamic integration methods. ${ }^{29,30}$ These methods are notorious for the heavy computational demand, however, since an explicit reference needs to be made to the intermediate states of the gradual variation process of the system. In this work, we 
develop an approximate approach to the solvation free energy of a solute molecule in a micellar solution on the basis of the method of energy representation. ${ }^{26-28}$ The method of energy representation is a theory of distribution functions in solution with strong emphasis on the solvation free energy. In the energy representation, the solute-solvent interaction energy is adopted for the coordinate of the distribution functions, and a functional for the solvation free energy is constructed in terms of energy distribution functions in the solution and reference solvent systems of interest. Since the set of configurations (structures) with the equal solute-solvent interaction energies are grouped into one at the outset, any approximation in the energy representation respects the statistical-mechanical principle that the configurations with the same solute-solvent interaction energies have equal weights in the solvation free energy. An approximate functional was presented in Refs. 27 and 28, with wellestablished performance for nonpolar, polar, and ionic solutes in water over a wide range of thermodynamic conditions. Actually, none of the system homogeneity, the thermodynamic limit, and the rigidity of molecule (and ion) is assumed in the formulation of the method of energy representation. A straightforward application of the method is thus possible to an inhomogeneous and partially finite system containing flexible species. In the practical implementation, the computation is required to be performed only at the initial and final states of the solute insertion process. The computational demand for the free-energy evaluation then becomes comparable to that for obtaining simple distribution functions in the systems of interest. ${ }^{31}$

In this work, we treat the solubilization of typical hydrophobic solutes in sodium dodecyl sulfate (SDS) micelle. Methane, benzene, and ethylbenzene are employed as the solutes, and their free energies of solvation are quantitatively discussed to assess the solubility change caused by the presence of a SDS micelle. To highlight the effect of the structure of the micellar aggregate, it is useful to conceptually divide the micellar system into the hydrophobic core, the headgroup region in contact with water, and the aqueous region outside the micelle. We evaluate the contribution from each region and show the extent of localization of the micelleinduced effect.

The organization of the paper is as follows. In Sec. II, a relationship is established between the free energy of solvation and the solubility change of a solute induced by micelle formation, and an approximate scheme is developed to evaluate the free energy in the micellar system. In Sec. III, the systems to be examined are identified and the computa- tional procedures are described. In Sec. IV, the solvation free energy is presented over a set of regions in the micellar system, and its difference between the micelle inside and bulk water is discussed for the quantitative and molecular-level evaluation of the solubilization.

\section{THEORY}

The purpose of this section is to construct an approximate expression for the solubility change of a solute induced by micelle formation. The construction consists of three steps. In Sec. II A, we present a general formulation for the solubility change and identify the solvation free energy to be evaluated. In Sec. II B, we introduce a scheme to highlight the presence of a micelle by dividing the micellar system into a set of regions. In Sec. II C, we describe the method of energy representation in an inhomogeneous and partially finite, mixed solvent system and provide an approximation to the solvation free energy in a micellar solution.

\section{A. Solubility change induced by micelle formation}

The system of our interest is a dilute solution containing a single solute molecule. The intermolecular interaction is supposed to be pairwise additive, and the formulation is based on the classical statistical mechanics. In the present treatment, a micellar solution is viewed as a mixed solvent system. The system involves the solvent water and the surfactant species forming a micellar aggregate. The other component may be the counterions, the surfactant species at the monomer state, and the cosolvents if prepared in the system. In the following developments, the micelle is assumed to be monodispersed, while the effect of polydispersity can be incorporated straightforwardly. The solute is also assumed to be dilute at saturation. In this case, the Boltzmann factor of the solvation free energy is the solubility expressed in the form of Ostwald coefficient. ${ }^{32}$

The solvation free energy is the free-energy change corresponding to the gradual insertion process of the solute molecule. In this free-energy change, only the contribution from the potential energy is involved and the ideal (kinetic) contribution is excluded at the outset. We let $\psi$ be the configuration of the solute molecule and $\mathbf{x}_{i, k}$ be the configuration of the $i$ th molecule of the $k$ th solvent species. The configuration is the collective denotation of the position and orientation and of the intramolecular degrees of freedom if the molecule is flexible. When the intermolecular interaction potential between the solute and the $k$ th solvent species is $v_{k}$, the solvation free energy $\Delta \mu_{m}$ in the micellar solution is expressed as

$$
\exp \left(-\beta \Delta \mu_{m}\right)=\frac{\int d V d \psi d \mathbf{X} \exp \left(-\beta\left\{\Psi(\psi)+\sum_{i, k} v_{k}\left(\psi, \mathbf{x}_{i, k}\right)+U(\mathbf{X})+P V\right\}\right)}{\int d V d \psi d \mathbf{X} \exp (-\beta\{\Psi(\psi)+U(\mathbf{X})+P V\})}
$$

where $\mathbf{X}$ represents the solvent configuration collectively, $U(\mathbf{X})$ is the total energy among the solvent, $\Psi(\psi)$ is the one-body energy of the solute, $P$ and $V$ are the pressure and system volume, respectively, and $\beta$ is the inverse of $k_{B} T$ with the Boltzmann constant $k_{B}$ and the temperature $T$. In Eq. (1), we adopt the isothermal-isobaric ensemble. This is because the solubility change upon micelle formation is typically examined in the isobaric condition, rather than in the isochoric. To attain the direct 
connection to the common practice of experiment, the solvation free energies in the micellar solution and in the neat solvent (pure water) are desirable to be evaluated at the same pressure (and temperature). ${ }^{33}$

In the following treatment, the micelle is supposed to be dilute. The supposition is certainly valid when the surfactant concentration is just above the critical micelle concentration. ${ }^{34}$ In this case, only one micellar aggregate needs to be treated explicitly and its center can be set to the origin of the system. Equation (1) is then rewritten as

$$
\exp \left(-\beta \Delta \mu_{m}\right)=\frac{\int^{*} d V d \psi d \mathbf{X} V \exp \left(-\beta\left\{\Psi(\psi)+\sum_{i, k} v_{k}\left(\psi, \mathbf{x}_{i, k}\right)+U(\mathbf{X})+P V\right\}\right)}{\int^{*} d V d \psi d \mathbf{X} V \exp (-\beta\{\Psi(\psi)+U(\mathbf{X})+P V\})},
$$

where $\int^{*}$ expresses the restriction that the origin of the position coordinate is the micellar center. A factor $V$ is involved in Eq. (2) since the center of the micelle is fixed. Of course, a restriction of the position of the micelle or solute is absent in Eq. (1). Within Eq. (2), the surfactants are localized near the origin, and the number is finite and equal to the aggregation number of the micelle. The surfactant molecules at the monomer state can be safely neglected in Eq. (2) when the critical micelle concentration is not too high. An explicit treatment is necessary for the counterions which may (partially) neutralize the micelle. They can be present at the micellar surface and interact with the solute molecule solubilized. The mixed solvent system in Eq. (2) thus consists of water, the surfactant molecules forming the micelle, and the counterions if the surfactant is ionic. ${ }^{35,36}$

When the solvent is neat (pure water), the corresponding free energy $\Delta \mu_{w}$ of solvation is given by

$$
\exp \left(-\beta \Delta \mu_{w}\right)=\frac{\int^{\#} d V d \psi d \mathbf{X} V \exp \left(-\beta\left\{\Psi(\psi)+\sum_{i} v_{1}\left(\psi, \mathbf{x}_{i, 1}\right)+U_{w}(\mathbf{X})+P V\right\}\right)}{\int^{\#} d V d \psi d \mathbf{X} V \exp \left(-\beta\left\{\Psi(\psi)+U_{w}(\mathbf{X})+P V\right\}\right)},
$$

where $\mathbf{X}$ represents the solvent configuration collectively, $U_{w}(\mathbf{X})$ is the total energy among the solvent (water), $v_{1}$ is the solute-water interaction potential, $\mathbf{x}_{i, 1}$ is the configuration of the $i$ th water molecule, and $\int^{\#}$ denotes the restriction that the position of the solute is fixed at the (arbitrarily chosen) origin of the system. A factor $V$ in Eq. (3) arises due to this restriction. Of course, the pure solvent is homogeneous in Eq. (3) and the inhomogeneity is introduced only after the solute insertion. Since $\Delta \mu_{w}$ determines the solubility of the solute without the micellar aggregate, it sets the reference for the solubility change due to the micelle formation. For the following development, we further introduce an auxiliary free-energy change $\Delta \tilde{\mu}_{w}$ as

$$
\exp \left(-\beta \Delta \tilde{\mu}_{w}\right)=\frac{\int^{\#} d V d \psi d \mathbf{X} V^{2} \exp \left(-\beta\left\{\Psi(\psi)+\Sigma_{i} v_{1}\left(\psi, \mathbf{x}_{i, 1}\right)+U_{w}(\mathbf{X})+P V\right\}\right)}{\int^{\#} d V d \psi d \mathbf{X} V^{2} \exp \left(-\beta\left\{\Psi(\psi)+U_{w}(\mathbf{X})+P V\right\}\right)}
$$

The difference between $\Delta \mu_{w}$ and $\Delta \tilde{\mu}_{w}$ is vanishingly small in the limit of large system size (thermodynamic limit) and is expressed as

$$
\Delta \tilde{\mu}_{w}-\Delta \mu_{w}=-k_{B} T \frac{\Delta V_{s}}{V_{0}}+o\left(\frac{1}{V_{0}}\right),
$$

where $\Delta V_{s}$ is the excess partial molar volume of the solute (the volume change upon insertion of the solute at a fixed position), $V_{0}$ is the average volume of the system of interest, and $o\left(1 / V_{0}\right)$ denotes a variable which vanishes faster than $1 / V_{0}$ in the thermodynamic limit $\left(V_{0} \rightarrow \infty\right) .{ }^{37}$ Although the distinction between $\Delta \mu_{w}$ and $\Delta \tilde{\mu}_{w}$ is of first order in $1 / V_{0}$ and is not necessary in the treatment of a property defined over a finite region, it becomes important for a property obtained through integration over the whole system, as seen below.

To treat the solubility change of the solute, we let $C_{m}$ be the concentration of the micellar aggregate and $S\left(C_{m}\right)$ be the solubility at the concentration of $C_{m}$. Of course, $S(0)$ is the solubility in the neat solvent (pure water), and a convention is adopted that $C_{m}$ and $S$ are expressed in the molarity scale. With $C_{m}$ and $S$, we introduce a measure $K_{m}$ of the solubility change due to the micelle formation through

$$
K_{m}=\lim _{C_{m} \rightarrow 0} \frac{1}{C_{m}}\left(\frac{S\left(C_{m}\right)}{S(0)}-1\right),
$$

where the (total) chemical potential of the solute is held fixed in the limiting procedure for $C_{m}{ }^{38} K_{m}$ is directly related to commonly used measures of the extent of solubilization. The solubilization capacity and the micelle-water partition coefficient in the mole fraction scale are equal to $S(0) K_{m} / n_{A}$ and $C_{w}\left(K_{m}+V_{m}\right) /\left(n_{A}+S(0) K_{m}+S(0) V_{m}\right)$, respectively, where $n_{A}$ is the aggregation number of the micelle, $C_{w}$ is the water density in molarity, and $V_{m}$ is the partial molar volume of the micellar aggregate. ${ }^{1-3,39,40}$ It is then seen in the developments of the Kirkwood-Buff theory ${ }^{32,41,42}$ that

$$
K_{m}=G_{s m}+\Delta V_{s}
$$

holds at low enough concentrations of the solute and the micellar aggregate. In this equation, $G_{s m}$ is the Kirkwood- 
Buff integral between the solute and the micelle and is defined as

$$
G_{s m}=\int d \mathbf{x}(g(\mathbf{x})-1),
$$

where $\mathbf{x}$ represents collectively the relative configuration between the solute and the micellar aggregate and $g(\mathbf{x})$ is the solute-micelle pair correlation function. ${ }^{41,43}$ Equations (7) and (8) show that although $K_{m}$ is directly connected to observables, $G_{s m}$ captures the micelle-induced effect more clearly. Indeed, the excess partial molar volume $\Delta V_{s}$ of the solute does not reflect any information about the micelle at dilute conditions.

In the Appendix, we show that

$$
K_{m}=-V_{0} \beta\left(\Delta \mu_{m}-\Delta \mu_{w}\right)+o(1),
$$

where $V_{0}$ is the (average) volume of the system and $o(1)$ denotes a variable which vanishes in the thermodynamic limit. ${ }^{44}$ Evidently, $K_{m}$ is determined by the difference between the solvation free energies with and without the micelle. Actually, the validity of Eq. (9) is not restricted to the case that the equilibrium is kept between the solution system of interest and the dilute gas phase. Equation (9) holds even when the counterpart of the equilibrium is solid or another solution. As far as the counterpart is common to the micellar solution and neat solvent, its contribution is canceled in Eq. (6). In fact, this is the reason why the ratio of the solubility is adopted in Eq. (6), rather than the difference.

As shown in the Appendix, the Kirkwood-Buff integral $G_{s m}$ is related to the free energy of solvation through

$$
G_{s m}=-V_{0} \beta\left(\Delta \mu_{m}-\Delta \tilde{\mu}_{w}\right)+o(1),
$$

where $\Delta \tilde{\mu}_{w}$ is the auxiliary free-energy change introduced by Eq. (4). ${ }^{44}$ Due to the presence of the factor $V_{0}$, the difference between $\Delta \mu_{w}$ and $\Delta \tilde{\mu}_{w}$ causes the distinction between $K_{m}$ and $G_{s m}$. Typically, $\Delta V_{s}$ is less than $\sim 10^{-1} 1 / \mathrm{mol}$ for small molecules. ${ }^{32}$ The $\Delta V_{s}$ term then affects the solubility change by a factor of $\sim 0.1$ with the micelle formation at the unit concentration $(1 \mathrm{~mol} / \mathrm{l})$. It is shown in Sec. IV, on the other hand, that $G_{s m}$ amounts to more than $\sim 10^{2} 1 /$ mol for model hydrophobic solutes in a micellar solution of sodium dodecyl sulfate. Thus, $K_{m}$ can be practically identified with $G_{s m}$ when the solubilization is effective.

\section{B. Division of the micellar system}

To approach the solubilization on the basis of the Kirkwood-Buff integral $G_{s m}$ given by Eq. (10), it is insightful to highlight the presence of a micelle by dividing the system into a set of regions. The emphasis needs to be placed on the region within or close to the micelle since only a single micelle is contained around the system origin and its effect diminishes in distant regions. A typical division of the system is into the hydrophobic core, the head-group region in contact with water, and the aqueous region outside the micelle. Of course, the division is not unique, and reflects the scheme of perceiving the system of interest.

We let $\Theta_{h}$ be the characteristic function for the $h$ th region. It is unity when the solute molecule is inside the region, and vanishes when the solute is outside. Since the division is made over the whole region of the solution,

$$
\sum_{h} \Theta_{h}(\psi, \mathbf{X})=1
$$

holds for each configuration $\psi$ of the solute and (collective) configuration $\mathbf{X}$ of the solvent. The solvation free energy $\Delta \mu_{m h}$ conditioned by the characteristic function $\Theta_{h}$ is then defined as

$$
\exp \left(-\beta \Delta \mu_{m h}\right)=\frac{\int^{*} d V d \psi d \mathbf{X} \Theta_{h}(\psi, \mathbf{X}) V \exp \left(-\beta\left\{\Psi(\psi)+\sum_{i, k} v_{k}\left(\psi, \mathbf{x}_{i, k}\right)+U(\mathbf{X})+P V\right\}\right)}{\int^{*} d V d \psi d \mathbf{X} \Theta_{h}(\psi, \mathbf{X}) V \exp (-\beta\{\Psi(\psi)+U(\mathbf{X})+P V\})},
$$

where $\int^{*}$ expresses the restriction given for Eq. (2) that the micellar center is fixed at the origin of the system. When $\Theta_{h}$ specifies the micellar interior or interface, $\Delta \mu_{m h}$ will be significantly different from the auxiliary free energy $\Delta \tilde{\mu}_{w}$ of solvation in the neat solvent (pure water) given by Eq. (4) and make the most important contribution to the solubilization. When the region characterized by $\Theta_{h}$ becomes more distant from the micelle, the difference between $\Delta \mu_{m h}$ and $\Delta \tilde{\mu}_{w}$ is expected to be smaller in magnitude and make a less important contribution.

The conditional free energy $\Delta \mu_{m h}$ of solvation is related to the (total) solvation free energy $\Delta \mu_{m}$ through

$$
\exp \left(-\beta \Delta \mu_{m}\right)=\sum_{h} p_{h} \exp \left(-\beta \Delta \mu_{m h}\right)
$$

where $p_{h}$ is given by

$$
p_{h}=\frac{\int^{*} d V d \psi d \mathbf{X} \Theta_{h}(\psi, \mathbf{X}) V \exp (-\beta\{\Psi(\psi)+U(\mathbf{X})+P V\})}{\int^{*} d V d \psi d \mathbf{X} V \exp (-\beta\{\Psi(\psi)+U(\mathbf{X})+P V\})} .
$$

Correspondingly, the Kirkwood-Buff integral $G_{s m}$ expressed as Eq. (10) is written exactly as

$$
G_{s m}=\sum_{h} v_{h}\left(\exp \left(-\beta\left(\Delta \mu_{m h}-\Delta \tilde{\mu}_{w}\right)\right)-1\right)
$$

in the limit of large system size $\left(V_{0} \rightarrow \infty\right)$, where $V_{0}$ is the (average) volume of the whole system and ${ }^{44,45}$

$$
v_{h}=V_{0} p_{h} .
$$

According to Eq. (14), $p_{h}$ is the probability that the solute is found in the region characterized by $\Theta_{h}$ when it is randomly inserted into the mixed solvent system as a test particle. $v_{h}$ is 
then equal to the (average) volume of that region, and sets a weight of geometrical origin in the sum of Eq. (15). ${ }^{46}$ Actually, when the volume $v_{h}$ remains finite in the limit of large system size, $\Delta \tilde{\mu}_{w}$ constituting the $h$ th term can be safely replaced by $\Delta \mu_{w}{ }^{47}$ Indeed, the difference between $\Delta \tilde{\mu}_{w}$ and $\Delta \mu_{w}$ vanishes in the thermodynamic limit and is irrelevant in the term corresponding to a finite region. It should be noted that $\exp \left(-\beta\left(\Delta \mu_{m h}-\Delta \tilde{\mu}_{w}\right)\right)$ is equal to the ratio of the average concentration of the solute in the region characterized by $\Theta_{h}$ to that at a position far from the micelle (in the bulk). Equation (15) is thus a discrete form of Kirkwood-Buff integral. On the basis of Eq. (15), an approximation to $G_{s m}$ is devised by retaining only the leading terms in the sum over $h$ and/or adopting an approximate method for $\Delta \mu_{m h}$ (and $\Delta \mu_{w}$ ).

In the Appendix, we show that the difference between $\Delta \mu_{m h}$ and $\Delta \widetilde{\mu}_{w}$ is of order $o\left(1 / V_{0}\right)$ when the region characterized by $\Theta_{h}$ corresponds to the limit of large distance from the micelle. The contribution from that region to $G_{s m}$ can then be neglected since the volume of the region is of order $O\left(V_{0}\right)$ in size. This means that the nonlocal component in the sense given in Ref. 48 is absent in the expression of Eq. (15) and that only the local contribution around the micelle needs to be taken into account to evaluate $G_{s m} \cdot{ }^{48,49}$ Actually, an expression similar to Eq. (15) holds also for $K_{m}$ by replacing $\Delta \tilde{\mu}_{w}$ with $\Delta \mu_{w}{ }^{47}$ The difference between $\Delta \mu_{m h}$ and $\Delta \mu_{w}$ is of first order in $1 / V_{0}$, however, in the region corresponding to the limit of large distance from the micelle. Thus, a nonvanishing contribution to $K_{m}$ in the thermodynamic limit is still present from the region far from the micelle and $K_{m}$ is not suitable to probe the local response around the micelle.

When the micellar system is divided into a set of regions, the leading contribution to the solubilization is expected from the micellar interior and/or interface. The approximation meeting this expectation is to retain the terms corresponding to the micellar interior and/or interface region in Eq. (15). The validity of such as approximation can be assessed within the line of solvation shell analysis ${ }^{48,50,51}$ by examining the convergence behavior of the summation of the partial contribution from each region. In Sec. IV, we show for model hydrophobic solutes in a micellar solution of sodium dodecyl sulfate that the contribution to the KirkwoodBuff integral $G_{s m}$ is the most important from the hydrophobic core and is negligible from the aqueous region outside the micelle.

\section{Approximation to the solvation free energy}

To approximately evaluate the solvation free energy $\Delta \mu_{m h}$ defined by Eq. (12), we employ the method of energy representation. The energy representation is introduced by adopting the value of the solute-solvent interaction of interest as the coordinate for the distribution of the solvent molecule around the solute. For a mixed solvent system, the energy coordinate is assigned to each solvent species. The coordinate for the $k$ th solvent species is denoted by $\epsilon_{k}$ and the corresponding instantaneous distribution $\hat{\rho}_{k}^{e}$ is defined as

$$
\hat{\rho}_{k}^{e}\left(\epsilon_{k}\right)=\sum_{i} \delta\left(v_{k}\left(\psi, \mathbf{x}_{i, k}\right)-\epsilon_{k}\right)
$$

where $v_{k}$ is the interaction potential between the solute and the $k$ th solvent species, $\mathbf{x}_{i, k}$ is the configuration of the $i$ th molecule of the $k$ th species relative to the solute, and the sum is taken over all the $k$ th solvent molecules. A superscript $e$ is attached to emphasize that a function is represented over the energy coordinate. The definition is of course parallel to that given in Refs. 26-28, and the distribution functions in the energy representation are generated from the averages of products of $\hat{\rho}_{k}^{e}$ in the system of interest. Even when the system involves more than one solvent species, the one-to-one correspondence is valid between the set of solute-solvent interaction potential functions and the set of one-body distribution functions in the energy representation. With the introduction of the energy coordinate for each solvent species, the relevant solute-solvent interaction is an array of functions whose $k$ th component is defined over the $k$ th coordinate $\epsilon_{k}$. The distribution function of concern is an array of energy distributions whose $k$ th component is the average of $\hat{\rho}_{k}^{e}$ under a given (array of) solute-solvent interaction potential function. The one-to-one correspondence can then be proved in the energy representation through a procedure similar to that given in Refs. 26 and $28 .^{52}$

To present an approximate expression for $\Delta \mu_{m h}$, it is convenient to introduce a notational convention for the average of a quantity $Q$ in the systems of interest. Two systems are actually of interest and are explicitly treated. In one of the systems, the solute is physically present and the solutesolvent interaction affects the probability distribution of the system configuration. The ensemble average is then denoted by $\langle Q\rangle_{m h}$ and is expressed as

$$
\langle Q\rangle_{m h}=\frac{\int^{*} d V d \psi d \mathbf{X} \Theta_{h}(\psi, \mathbf{X}) V Q \exp \left(-\beta\left\{\Psi(\psi)+\sum_{i, k} v_{k}\left(\psi, \mathbf{x}_{i, k}\right)+U(\mathbf{X})+P V\right\}\right)}{\int^{*} d V d \psi d \mathbf{X} \Theta_{h}(\psi, \mathbf{X}) V \exp \left(-\beta\left\{\Psi(\psi)+\sum_{i, k} v_{k}\left(\psi, \mathbf{x}_{i, k}\right)+U(\mathbf{X})+P V\right\}\right)} .
$$

In the other, the solute is placed as a test particle and its configuration is uncoupled from the solvent configuration. The average is written as $\langle Q\rangle_{m h, 0}$ and is given by

$$
\langle Q\rangle_{m h, 0}=\frac{\int^{*} d V d \psi d \mathbf{X} \Theta_{h}(\psi, \mathbf{X}) V Q \exp (-\beta\{\Psi(\psi)+U(\mathbf{X})+P V\})}{\int^{*} d V d \psi d \mathbf{X} \Theta_{h}(\psi, \mathbf{X}) V \exp (-\beta\{\Psi(\psi)+U(\mathbf{X})+P V\})} .
$$


The evaluation through Eq. (19) needs only the ensemble for the mixed solvent system in which no interaction is physically present between the solute and solvent molecules. The solute is a test particle and is to be inserted according to the probability distribution of the configuration $\psi$ generated by the one-body energy $\Psi(\psi)$.

The formulation of an approximate functional for the solvation free energy $\Delta \mu_{m h}$ is then parallel to that described in Ref. 27. According to the approximations adopted in Ref. $27, \Delta \mu_{m h}$ is given by a set of definitions and equations listed as

$$
\begin{aligned}
& \rho_{k}^{e}\left(\epsilon_{k}\right)=\left\langle\hat{\rho}_{k}^{e}\left(\epsilon_{k}\right)\right\rangle_{m h}, \\
& \rho_{k, 0}^{e}\left(\epsilon_{k}\right)=\left\langle\hat{\rho}_{k}^{e}\left(\epsilon_{k}\right)\right\rangle_{m h, 0}, \\
& \chi_{k l, 0}^{e}\left(\epsilon_{k}, \eta_{l}\right)=\left\langle\hat{\rho}_{k}^{e}\left(\epsilon_{k}\right) \hat{\rho}_{l}^{e}\left(\eta_{l}\right)\right\rangle_{m h, 0}-\left\langle\hat{\rho}_{k}^{e}\left(\epsilon_{k}\right)\right\rangle_{m h, 0}\left\langle\hat{\rho}_{l}^{e}\left(\eta_{l}\right)\right\rangle_{m h, 0},
\end{aligned}
$$

$$
\begin{aligned}
w_{k}^{e}\left(\epsilon_{k}\right)= & -k_{B} T \log \left(\frac{\rho_{k}^{e}\left(\epsilon_{k}\right)}{\rho_{k, 0}^{e}\left(\epsilon_{k}\right)}\right)-\epsilon_{k}, \\
w_{k, 0}^{e}\left(\epsilon_{k}\right)= & -k_{B} T \sum_{l} \int d \eta_{l}\left(\frac{\delta_{k l} \delta\left(\epsilon_{k}-\eta_{l}\right)}{\rho_{k, 0}^{e}\left(\epsilon_{k}\right)}\right. \\
& \left.\left.-\left(\chi_{k l, 0}^{e}\right)^{-1}\left(\epsilon_{k}, \eta_{l}\right)\right)\left(\rho_{l}^{e}\left(\eta_{l}\right)-\rho_{l, 0}^{e}\left(\eta_{l}\right)\right)\right),
\end{aligned}
$$

$$
\begin{aligned}
\Delta \mu= & -k_{B} T \sum_{k} \int d \epsilon_{k}\left[\left(\rho_{k}^{e}\left(\epsilon_{k}\right)-\rho_{k, 0}^{e}\left(\epsilon_{k}\right)\right)+\beta w_{k}^{e}\left(\epsilon_{k}\right) \rho_{k}^{e}\left(\epsilon_{k}\right)\right. \\
& -\left\{\alpha_{k}\left(\epsilon_{k}\right) F_{k}\left(\epsilon_{k}\right)+\left(1-\alpha_{k}\left(\epsilon_{k}\right)\right) F_{k, 0}\left(\epsilon_{k}\right)\right\}\left(\rho_{k}^{e}\left(\epsilon_{k}\right)\right. \\
& \left.\left.-\rho_{k, 0}^{e}\left(\epsilon_{k}\right)\right)\right]
\end{aligned}
$$

Since the formulation is generally applicable to a mixed solvent system, the subscript $m h$ is omitted for $\rho_{k}^{e}, \rho_{k, 0}^{e}, \chi_{k l, 0}^{e}, w_{k}^{e}$, $w_{k, 0}^{e}, \Delta \mu, F_{k}, F_{k, 0}$, and $\alpha_{k}$. In Eq. (24), $\left(\chi_{k 3}^{e}{ }_{k l, 0}\right)^{-1}$ is the inverse of the correlation matrix $\chi_{k l, 0}^{e}$ satisfying ${ }^{53}$

$$
\sum_{n} \int d \xi_{n} \chi_{k n, 0}^{e}\left(\epsilon_{k}, \xi_{n}\right)\left(\chi_{n l, 0}^{e}\right)^{-1}\left(\xi_{n}, \eta_{l}\right)=\delta_{k l} \delta\left(\epsilon_{k}-\eta_{l}\right)
$$

It should be noted in Eqs. (20)-(28) that the solvation free energy $\Delta \mu$ is constructed from the energy distribution functions $\rho_{k}^{e}, \rho_{k, 0}^{e}$, and $\chi_{k l, 0}^{e}$ defined by Eqs. (20)-(22), respectively. These distribution functions are the inputs to the approximate functional for $\Delta \mu$ and are obtained from the two simulations corresponding to Eqs. (18) and (19).

When the solute molecule is flexible and its (intramolecular) structure varies significantly with the solvation, the approximate scheme given by Eqs. (20)-(28) may not perform well in the original form. In this case, a modified form of functional is needed for the solvation free energy. The formulation of the modification is described in Ref. 28 and its extension to a mixed solvent system is straightforward.
In this section, the free energy of binding of a molecule to micelle is approximately treated by viewing the micellar solution as an inhomogeneous and partially finite, mixed solvent system. A natural fluctuation according to the potential functions is allowed for the configurations of the micelle and its constituent surfactant. Our development is possible because none of the system homogeneity, the thermodynamic limit, and the rigidity of molecule or ion is assumed in the method of energy representation. Thus, the treatment is not restricted to the solubilization in micelle, and will be equally applicable to the binding of a molecule (ligand) to such nanoscale structures as protein and membrane.

\section{COMPUTATIONAL PROCEDURES}

In the present work, the surfactant is sodium dodecyl sulfate (SDS). The structure of the dodecyl sulfate anion $\left(\mathrm{CH}_{3}\left(\mathrm{CH}_{2}\right)_{11} \mathrm{OSO}_{3}^{-1}\right)$ is illustrated in Fig. 1. The united-atom approximation was adopted for the methyl and methylene groups, and the hydrogen atoms were not explicitly treated. The bond lengths and angles are fixed. Their values were 


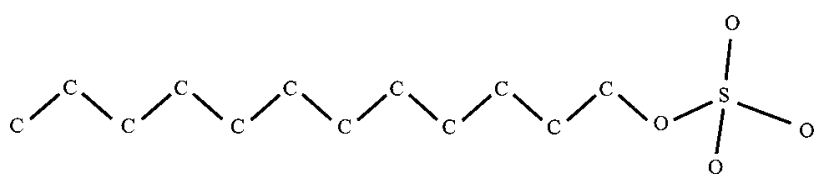

FIG. 1. The structure of the dodecyl sulfate anion $\left(\mathrm{CH}_{3}\left(\mathrm{CH}_{2}\right)_{11} \mathrm{OSO}_{3}^{-1}\right)$. The united-atom approximation is adopted for the methyl and methylene groups, and the hydrogen atoms are not shown.

taken from Refs. 11 and 54, and are listed in Table I. Each of $\mathrm{C}-\mathrm{C}-\mathrm{C}-\mathrm{C}, \mathrm{C}-\mathrm{C}-\mathrm{C}-\mathrm{O}, \mathrm{C}-\mathrm{C}-\mathrm{O}-\mathrm{S}$, and $\mathrm{C}-\mathrm{O}-\mathrm{S}-\mathrm{O}$ in Fig. 1 was subject to the torsional motion. The Ryckaert-Bellemans potential in the form of

$$
\sum_{i=0}^{5} C_{i}(\cos (\varphi))^{i}
$$

was used for the $\mathrm{C}-\mathrm{C}-\mathrm{C}-\mathrm{C}$ torsion, where $\varphi$ is the dihedral angle with $\varphi=0$ at the trans conformation and $C_{0^{-}} C_{5}$ are $2.22, \quad 2.91,-3.14,-0.73,6.27$, and $-7.53 \mathrm{kcal} / \mathrm{mol}$, respectively. ${ }^{55}$ For the dihedral-angle degrees of freedom involving the head-group atoms $(\mathrm{C}-\mathrm{C}-\mathrm{C}-\mathrm{O}, \mathrm{C}-\mathrm{C}-\mathrm{O}-\mathrm{S}$, and $\mathrm{C}-\mathrm{O}-\mathrm{S}-\mathrm{O}$ ), the AMBER form of potential ${ }^{56}$ was adopted with the parameters given in Table 1 of Ref. 57.

The solute molecules to be solubilized are methane, benzene, and ethylbenzene. The methane molecule and the methyl and methylene groups of ethylbenzene were described as united atoms, while the hydrogens attached to the phenyl rings of benzene and ethylbenzene were treated explicitly as interaction sites. The (intramolecular) structure was supposed to be rigid for all of the three solute molecules. The bond lengths and angles of benzene and ethylbenzene were obtained from Refs. 58 and 59 and are shown in Table I. For ethylbenzene, the interaction sites corresponding to the methyl and methylene groups were placed so that the dihedral angle formed by the methyl, methylene, and the two adjacent carbon sites is $90^{\circ}$ and that the molecule has a perpendicular structure.

The water molecule was treated as rigid and nonpolarizable, and the TIP3P model was adopted as the potential function. ${ }^{60}$ The sodium ion was set to be a nonpolarizable, single-site particle. The pairwise additivity was assumed, and the Lennard-Jones and Coulombic interactions were operative between a pair of sites belonging to different molecules and two sites separated by three or more bonds in the dodecyl sulfate anion. The potential parameters (Lennard-Jones $\epsilon$ and $\sigma$ and partial charges) for the SDS and solute molecules were taken from Refs. 11, 54, 58, 61, and 62 and are listed in Table I. The standard Lorentz-Berthelot combining rule was used to construct the Lennard-Jones part of the interaction between different sites. ${ }^{29}$ For the intramolecular interaction of the dodecyl sulfate anion, the Lennard-Jones and Coulombic interactions between two sites with three-bond separation were reduced by factors of 2.0 and 1.2 , respectively. ${ }^{63}$

The aggregation number of the micelle was set to 60 , a value close to the experiment. ${ }^{1-3,64}$ To simulate the micellar system, 60 dodecyl sulfate anions, 60 sodium cations, 10000 water molecules, and a single solute molecule were located in a cubic unit cell. For the solute position in the micelle, six regions with respect to the distance $r$ of the center of mass of the solute from that of the 60 dodecyl sulfate anions were examined by concentrically dividing the domain of $r$ $\leqslant 30 \AA$ with an equal interval of $5 \AA$. Two systems were explicitly treated for each $r$ region, as described in Sec. II C. To treat the system containing the solute physically and obtain the ensemble average in the form of Eq. (18), the restriction on the solute position was implemented with the potential given by

$$
\left\{\begin{array}{l}
\frac{K_{r}}{2}\left(\frac{r-r_{\min }}{r_{w}}\right)^{2}, \quad\left(\text { when } r<r_{\min }\right), \\
0, \quad\left(\text { when } r_{\min }<r<r_{\max }\right) \\
\frac{K_{r}}{2}\left(\frac{r-r_{\max }}{r_{w}}\right)^{2}, \quad\left(\text { when } r>r_{\max }\right),
\end{array}\right.
$$

where $r_{\min }$ and $r_{\max }$ are the lower and upper limits of the $r$ region of interest, respectively, and $K_{r}$ and $r_{w}$ were taken to be $20 \mathrm{kcal} / \mathrm{mol}$ and $0.2 \AA$, respectively. In this case, the system configuration was counted for the ensemble average only when $r_{\min }<r<r_{\max }$. When the simulated system involves the solute as a test particle and the ensemble average is expressed as Eq. (19), the solute was simply inserted with the condition of $r_{\min }<r<r_{\max }$ at random orientation.

The system was sampled with the molecular dynamics method in the isothermal-isobaric ensemble. The temperature and the pressure were set to $25{ }^{\circ} \mathrm{C}$ and $1 \mathrm{~atm}$, respectively. The Martyna-Tobias-Klein algorithm was adopted for the control of the temperature and pressure, ${ }^{65}$ and the particles and barostat were coupled to the same Nosé-Hoover chain of length $5{ }^{66}$ The characteristic frequency was taken to be 1.0 and $0.5 \mathrm{ps}^{-1}$ for the thermostat and barostat, respectively, and the masses of the thermostat and barostat were determined with the procedure recommended in Refs. 65 and 66. The equation of motion for the rigid body was integrated using the velocity Verlet algorithm ${ }^{30,67}$ for the translational part and a quarternion algorithm ${ }^{68}$ for the orientational part, and the RATTLE algorithm ${ }^{69}$ was employed for the flexible particle. The time step of integration was $2 \mathrm{fs}$, and the electrostatic potential was handled by the Ewald method with the surrounding medium of infinite dielectric constant. The energy distribution functions were constructed by averaging the instantaneous histogram given by Eq. (17), and each of the water molecule, sodium cation, dodecyl sulfate anion was treated as a distinct solvent species. The system containing the solute physically was simulated for $500 \mathrm{ps}$. The solutesolvent pair energy was sampled every $10 \mathrm{fs}$ and used to construct the energy distribution function. For the system involving the solute as a test particle, the simulation was performed for $500 \mathrm{ps}$. The (instantaneous) configuration of the mixed solvent system was sampled every $100 \mathrm{fs}$, and the solute insertion was performed 1000 times at each solvent configuration sampled. ${ }^{70}$

The simulation was also conducted for the solute molecule solvated in neat water. In this case, 10000 water molecules and a single solute molecule were located in the unit 
TABLE I. The bond lengths and angles, the Lennard-Jones $\epsilon$ and $\sigma$, and the partial charges for sodium cation, dodecyl sulfate anion, and solute molecules.

\begin{tabular}{|c|c|c|c|c|c|}
\hline Site or group & $\begin{array}{c}\text { Bond } \\
\text { length }(\AA)\end{array}$ & $\begin{array}{c}\text { Bond } \\
\text { angle (degree) }\end{array}$ & $\epsilon(\mathrm{kcal} / \mathrm{mol})$ & $\sigma(\AA)$ & $\begin{array}{l}\text { Partial } \\
\text { charge }\end{array}$ \\
\hline \multicolumn{6}{|l|}{ Dodecyl sulfate anion ${ }^{\mathrm{b}}$} \\
\hline $\mathrm{CH}_{3}$ & & & 0.175 & 3.905 & 0 \\
\hline $\mathrm{CH}_{2}$ (internal) & & & 0.118 & 3.905 & 0 \\
\hline $\mathrm{CH}_{2}$ (attached to $\left.\mathrm{O}\right)$ & & & 0.118 & 3.905 & 0.137 \\
\hline $\mathrm{O}($ ester$)$ & & & 0.170 & 3.000 & -0.459 \\
\hline S & & & 0.250 & 3.550 & 1.284 \\
\hline $\mathrm{O}\left(\mathrm{SO}_{3}\right)$ & & & 0.200 & 3.150 & -0.654 \\
\hline $\mathrm{C}-\mathrm{C}$ & 1.53 & & & & \\
\hline $\mathrm{C}-\mathrm{O}($ ester$)$ & 1.42 & & & & \\
\hline $\mathrm{O}$ (ester)-S & 1.58 & & & & \\
\hline $\mathrm{S}-\mathrm{O}\left(\mathrm{SO}_{3}\right)$ & 1.46 & & & & \\
\hline $\mathrm{C}-\mathrm{C}-\mathrm{C}$ & & $111.0^{\circ}$ & & & \\
\hline $\mathrm{C}-\mathrm{C}-\mathrm{O}$ (ester) & & $109.5^{\circ}$ & & & \\
\hline $\mathrm{C}-\mathrm{O}$ (ester)-S & & $112.6^{\circ}$ & & & \\
\hline $\mathrm{O}($ ester $)-\mathrm{S}-\mathrm{O}\left(\mathrm{SO}_{3}\right)$ & & $102.6^{\circ}$ & & & \\
\hline $\mathrm{O}\left(\mathrm{SO}_{3}\right)-\mathrm{S}-\mathrm{O}\left(\mathrm{SO}_{3}\right)$ & & $115.4^{\circ}$ & & & \\
\hline \multicolumn{6}{|l|}{ Sodium cation } \\
\hline $\mathrm{Na}^{+}$ & & & 0.115 & 2.275 & 1 \\
\hline \multicolumn{6}{|l|}{ Methane } \\
\hline $\mathrm{CH}_{4}$ & & & 0.294 & 3.730 & 0 \\
\hline \multicolumn{6}{|l|}{ Benzene } \\
\hline $\mathrm{C}$ & & & 0.07 & 3.55 & -0.115 \\
\hline $\mathrm{H}$ & & & 0.03 & 2.42 & 0.115 \\
\hline $\mathrm{C}-\mathrm{C}$ & 1.40 & & & & \\
\hline $\mathrm{C}-\mathrm{H}$ & 1.08 & & & & \\
\hline $\mathrm{C}-\mathrm{C}-\mathrm{C}$ & & $120.0^{\circ}$ & & & \\
\hline $\mathrm{C}-\mathrm{C}-\mathrm{H}$ & & $120.0^{\circ}$ & & & \\
\hline \multicolumn{6}{|l|}{ Ethylbenzene } \\
\hline $\mathrm{C}($ phenyl) & & & 0.07 & 3.55 & -0.115 \\
\hline $\mathrm{H}($ phenyl) & & & 0.03 & 2.42 & 0.115 \\
\hline $\mathrm{CH}_{3}$ & & & 0.175 & 3.905 & 0 \\
\hline $\mathrm{CH}_{2}$ & & & 0.118 & 3.905 & 0.115 \\
\hline $\mathrm{C}$ (phenyl)-C(phenyl) & 1.40 & & & & \\
\hline $\mathrm{C}$ (phenyl)-H(phenyl) & 1.08 & & & & \\
\hline $\mathrm{C}($ phenyl $)-\mathrm{C}\left(\mathrm{CH}_{2}\right)$ & 1.51 & & & & \\
\hline $\mathrm{C}\left(\mathrm{CH}_{2}\right)-\mathrm{C}\left(\mathrm{CH}_{3}\right)$ & 1.53 & & & & \\
\hline $\mathrm{C}$ (phenyl)-C(phenyl)-C(phenyl) & & $120.0^{\circ}$ & & & \\
\hline $\mathrm{C}($ phenyl)-C(phenyl)-H(phenyl) & & $120.0^{\circ}$ & & & \\
\hline $\mathrm{C}($ phenyl $)-\mathrm{C}($ phenyl $)-\mathrm{C}\left(\mathrm{CH}_{2}\right)$ & & $120.0^{\circ}$ & & & \\
\hline $\mathrm{C}($ phenyl $)-\mathrm{C}\left(\mathrm{CH}_{2}\right)-\mathrm{C}\left(\mathrm{CH}_{3}\right)$ & & $114.0^{\circ}$ & & & \\
\hline
\end{tabular}

${ }^{\mathrm{a}}$ The partial charge is expressed in the unit of elementary charge.

${ }^{\mathrm{b}}$ For the dodecyl sulfate anion, $\mathrm{O}$ (ester) denotes the oxygen site connected to both $\mathrm{C}$ and $\mathrm{S}$, and $\mathrm{O}\left(\mathrm{SO}_{3}\right)$ is the oxygen site connected only to $S$.

cell, and the system is homogeneous when the system involves the solute as a test particle. The interaction potential and the simulation condition and procedure were the same as those for the micellar system, except that the simulation length was 100 and 50 ps when the solute is treated as physical and test particles, respectively. ${ }^{70}$

\section{RESULTS AND DISCUSSION}

In Fig. 2, we show the densities of the hydrophobic tail, the head group, and water as functions of the distance $r$ from the micellar center identified as the center of mass of the 60 dodecyl sulfate anions. Since the eccentricity of sodium dodecyl sulfate micelle was found to be small,,${ }^{11,12,21}$ it is natural to divide the micellar system into a set of regions on the basis of Fig. 2. As described in Sec. III, we introduce six regions in terms of the center-of-mass distance $r$ of the solute from the micellar center by concentrically dividing the domain of $r \leqslant 30 \AA$ with an equal interval of $5 \AA$. In the following, the regions are numbered I, ..., VI from the micelle inside to outside. According to Fig. 2, regions I-III correspond to the hydrophobic core, where water is scarcely 


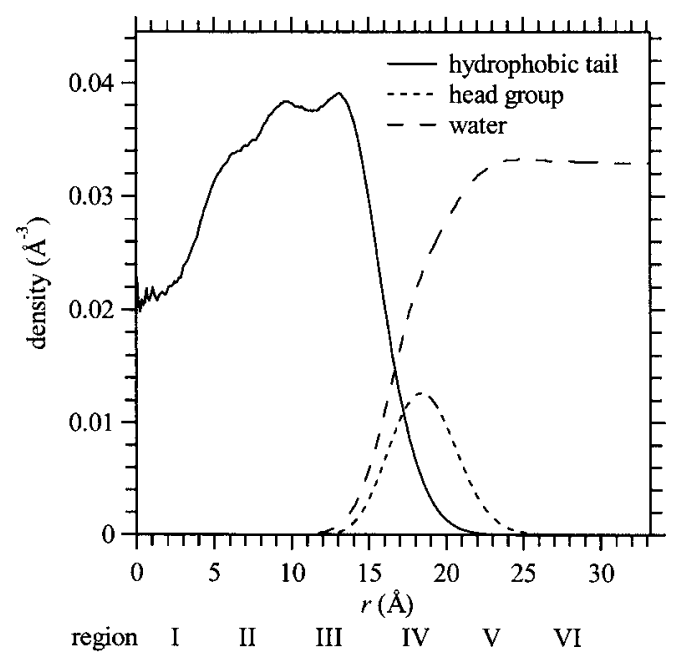

FIG. 2. The densities of the hydrophobic tail, the head group, and water as functions of the distance $r$ from the center of mass of the dodecyl sulfate anions forming the micelle. The density of the hydrophobic tail refers to the sum of the (number) densities of the methyl and methylene groups of the dodecyl sulfate anion, and the density of the head group is the sum (number) density of the sulfur and oxygen atoms. The water density is expressed with respect to the center of mass of the water molecule. Six regions are introduced by dividing the domain of $r \leqslant 30 \AA$ with an interval of $5 \AA$, and are numbered I,..., VI from the micelle inside to outside.

present. Region IV refers to the head-group region in contact with water, and is the transition region from the hydrophobic core to the aqueous region outside the micelle represented by regions $\mathrm{V}$ and $\mathrm{VI}{ }^{71}$

In Fig. 3, we show the solvation free energy $\Delta \mu$ of the solute in micellar regions I-VI and in bulk water. The bulk denotes the region far from the micelle, and $\Delta \mu$ in the bulk is the solvation free energy of the solute in neat water. It is evident in Fig. 3 that each of methane, benzene, and ethylbenzene is free-energetically stabilized in hydrophobic-core regions I-III. $\Delta \mu$ is not significantly different from the bulk value, on the other hand, in aqueous regions V-VI outside the micelle. A transition behavior is seen in head-group region IV. The $\Delta \mu$ reduction relative to the bulk value is present in the transition region, but is weaker than in the hydrophobic core. Figure 3 is thus a quantitative demonstration that the micelle inside is a favorable environment for hydrophobic solutes, in agreement with the conventional ideas $^{1-3}$ and a molecular simulation study of small nonpolar solutes in a nonionic micelle of octyl pentaethylene glycol ether $\left(\mathrm{C}_{8} \mathrm{E}_{5}\right){ }^{23}$

In our procedure described in Sec. II C, $\Delta \mu$ in Fig. 3 is directly connected to the distribution functions at the molecular level. Figure 4 illustrates the energy distribution functions $\rho_{k}^{e}$ and $\rho_{k, 0}^{e}$ of methane and benzene in region III and the bulk for the solute-water and solute-dodecyl sulfate anion pair interaction. Obviously, $\rho_{k}^{e}$ and $\rho_{k, 0}^{e}$ of the solute-water pair are more concentrated near $\epsilon=0$ in region III than in the bulk. Indeed, water is scarcely present in the hydrophobic core. When $\epsilon$ is large, $\rho_{k, 0}^{e}$ is smaller in the micelle inside, on the whole. This shows that the excluded volume effect is weaker within the micelle than in the bulk. In the attractive $\epsilon$ domain, $\rho_{k}^{e}$ and $\rho_{k, 0}^{e}$ are larger for dodecyl sulfate than for water. Actually, the favorable component of the benzene-

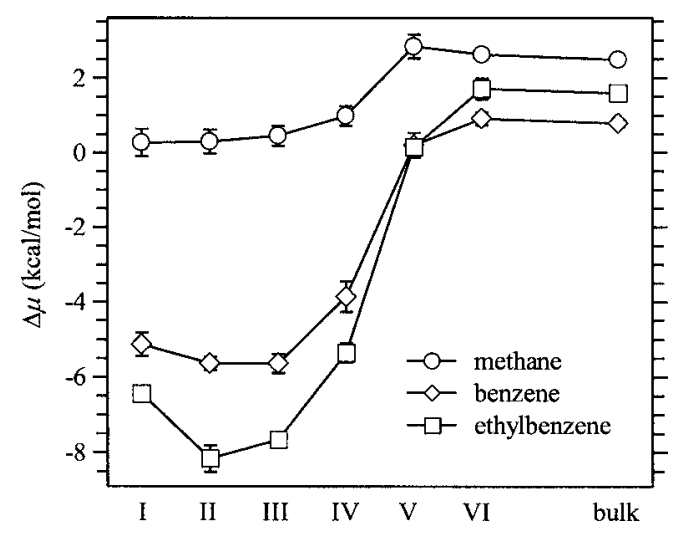

FIG. 3. The solvation free energy $\Delta \mu$ in micellar regions I-VI and in the bulk. Regions I-VI are numbered from the micelle inside to outside by identifying the solute position in terms of the center-of-mass distance $r$ from the micellar center and dividing the domain of $r \leqslant 30 \AA$ concentrically with an equal interval of $5 \AA$. The bulk denotes the region far from the micelle, and $\Delta \mu$ in the bulk is equal to the solvation free energy of the solute inserted into neat water. The error bar is expressed at the $95 \%$ confidence level, and is smaller than the size of the corresponding data symbol when it is not shown. The lines connecting the data are drawn for the eye guide.

dodecyl sulfate interaction is more than the thermal energy $\left(k_{B} T\right)$ by several times in magnitude. Thus, both the excluded volume effect and the dispersion attraction contribute positively to the micelle-induced enhancement of the solubility. When methane and benzene are compared, $\rho_{k}^{e}$ and $\rho_{k, 0}^{e}$ are larger for benzene in both region III and the bulk except when the energy coordinate $\epsilon$ is close to 0 . Benzene carries more interaction sites than methane, and the repulsive and attractive interactions are both stronger. The competition of the attractive interaction against the repulsive is more favorable for benzene than for methane. As seen in Fig. 3, the extent of stabilization in regions I-IV relative to the bulk is stronger for benzene and ethylbenzene than for methane.

According to Eq. (15) and Fig. 3, the Kirkwood-Buff integral $G_{s m}$ is estimated as $(4 \pm 1) \times 10^{2},(5 \pm 1) \times 10^{5}$, and $(7 \pm 2) \times 10^{7} 1 / \mathrm{mol}$ for methane, benzene, and ethylbenzene, respectively, where the sum over $h$ in Eq. (15) is taken over regions I-VI. ${ }^{40}$ As described with respect to Eq. (10), $G_{s m}$ is essentially equal to the measure $K_{m}$ of the solubility change given by Eq. (6) and represents the ability of the micelle to incorporate a hydrophobic solute. The common practice to analyze $G_{s m}$ (or $K_{m}$ ) in terms of the distinction between the micelle inside and the bulk water is the pseudophase model, in which the micellar portion of the system is viewed as a separate phase. ${ }^{1-3}$ In the pseudophase model, the aqueous region outside the micelle is considered unimportant and the hydrophobic core is typically assumed to make a dominant contribution in determining the extent of solubilization. To examine the importance of each region in the determination of $G_{s m}$ on the basis of Eq. (15), we introduce the relative contribution $P_{h}$ from the $h$ th region by

$$
P_{h}=\frac{v_{h}\left(\exp \left(-\beta\left(\Delta \mu_{m h}-\Delta \tilde{\mu}_{w}\right)\right)-1\right)}{G_{s m}},
$$

where the variables were defined in Sec. II. Of course, the sum of $P_{h}$ over $h=\mathrm{I}, \ldots, \mathrm{VI}$ is unity. In Fig. 5, we show $P_{h}$ over regions I-VI. Evidently, $G_{s m}$ (or $K_{m}$ ) is essentially de- 

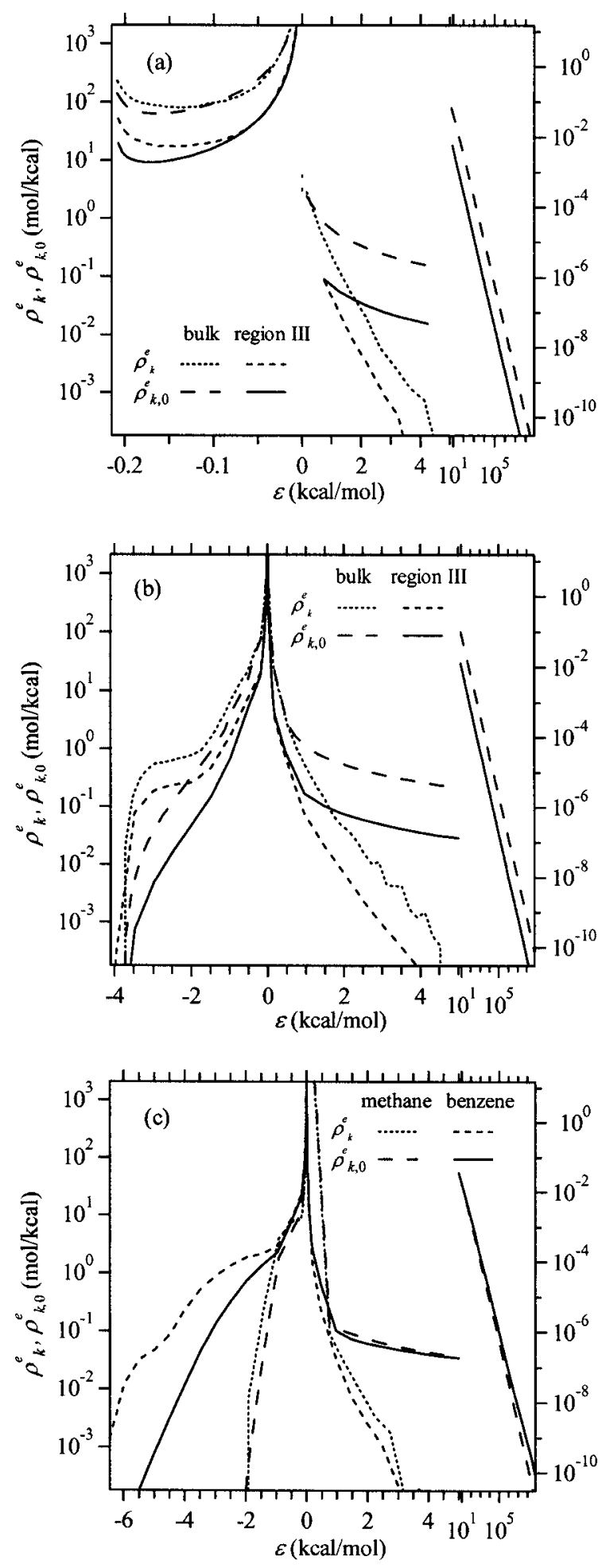

FIG. 4. The energy distribution functions $\rho_{k}^{e}$ and $\rho_{k, 0}^{e}$ against the solutesolvent interaction energy $\epsilon$ (a) for methane-water pair in region III and the bulk, (b) for benzene-water pair in region III and the bulk, and (c) for methane and benzene with dodecyl sulfate anion in region III. When $\epsilon$ $\leqslant 5 \mathrm{kcal} / \mathrm{mol}$, the abscissa is linearly graduated and the ordinate refers to the left. When $\epsilon \geqslant 5 \mathrm{kcal} / \mathrm{mol}$, the graduation for the abscissa is logarithmic and only $\rho_{k, 0}^{e}$ is shown with respect to the right ordinate. In (a), the graduation is also changed at $\epsilon=0$. The logarithmic graduation is adopted for both the left and right ordinates.

termined by the contributions from regions II-IV. Region I is not significant simply because its volume $\left[v_{h}\right.$ of Eq. (32)] is small. The importance of head-group region IV relative to

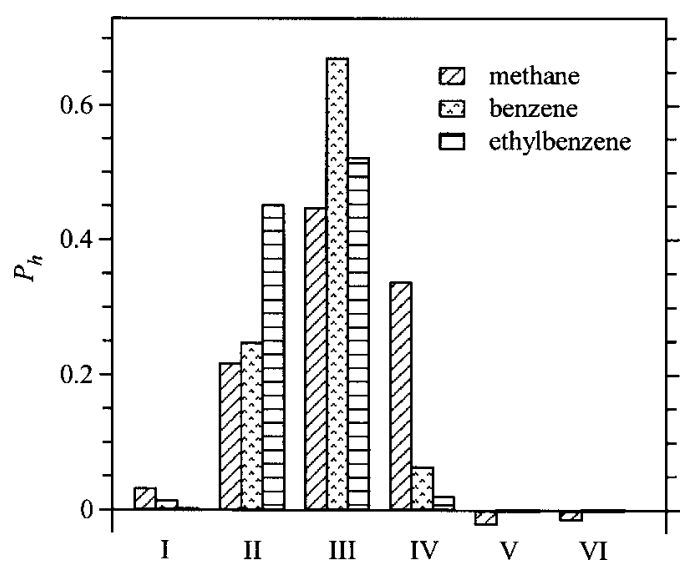

FIG. 5. The relative contribution $P_{h}$ from the $h$ th region $(h=\mathrm{I}, \ldots, \mathrm{VI})$ to the Kirkwood-Buff integral $G_{s m}$. The error of $P_{h}$ at the 95\% confidence level is $50 \%-70 \%$ of the $P_{h}$ value itself since the $\Delta \mu$ error is incorporated into Eq. (32) in the exponential form.

hydrophobic core II and III is appreciable for methane. In other words, the solubilization of benzene and ethylbenzene is more sharply characterized as a transfer from the aqueous to hydrophobic environment. For all the solutes examined, the contribution is negligible from aqueous regions $\mathrm{V}$ and VI outside the micelle. This is a support to the pseudophase model. The support is stronger for benzene and ethylbenzene since the hydrophobic-core region makes a dominant contribution.

Within the ordinate scale of Fig. 5, $P_{h}$ is hardly distinguishable from the probability of finding the solute in the $h$ th region among regions I-VI. ${ }^{72}$ The above description concerning $P_{h}$ can then be rephased in terms of the probability. Figure 5 shows that the solute is more localized in the hydrophobic core for benzene and ethylbenzene than for methane. The distribution within the hydrophobic interior is rather diffuse, in agreement with experimental reports of benzene in sodium dodecyl sulfate micelle. ${ }^{73,74}$

\section{ACKNOWLEDGMENTS}

This work is supported by the Grant-in-Aid for Scientific Research (No. 15205004) from the Japan Society for the Promotion of Science and by the Grant-in-Aid for Scientific Research on Priority Areas (No. 15076205), the Grant-in-Aid for Creative Scientific Research (No. 13NP0201), and the NAREGI (National Research Grid Initiative) Project from the Ministry of Education, Culture, Sports, Science, and Technology. One of the authors (N.M.) is also grateful to the Supercomputer Laboratory of Institute for Chemical Research, Kyoto University, for generous allocation of computation time.

\section{APPENDIX: PROOF OF EQS. (9) AND (10)}

In this appendix, we analyze the ensemble dependence of the averages involved in the Kirkwood-Buff integral and prove Eq. (10). Equation (9) simply follows from Eqs. (5), (7), and (10).

We start with an expression for the Kirkwood-Buff integral $G_{s m}$ in the grand canonical ensemble. Let $\hat{N}_{s}$ and $\hat{N}_{m}$ be 
the instantaneous numbers of the solute molecule and the micellar aggregate, respectively. The micellar aggregate is treated as a single particle with intra-aggregate degrees of freedom. $G_{s m}$ is then expressed as

$$
G_{s m}=V_{0}\left(\frac{\left\langle\hat{N}_{s} \hat{N}_{m}\right\rangle_{\mu V T}}{\left\langle\hat{N}_{s}\right\rangle_{\mu V T}\left\langle\hat{N}_{m}\right\rangle_{\mu V T}}-1\right),
$$

where $\langle\cdots\rangle_{\mu V T}$ denotes the ensemble average in the grand canonical ensemble and $V_{0}$ is the volume of the system. ${ }^{41,44}$

The next step is to transform Eq. (A1) into a form similar to Eq. (2). To do so, it is notationally convenient to introduce a variable $Z$ as

$$
Z=\int d \psi \exp (-\beta\{\Psi(\psi)+W(\psi, \mathbf{X})\}),
$$

where $\psi$ is the configuration of the solute, $\Psi(\psi)$ is the onebody energy of the solute, $\mathbf{X}$ represents the solvent configuration collectively, and $W(\psi, \mathbf{X})$ is the sum of the interaction energy between the solute and solvent molecules. The translational degrees of freedom of the solute is incorporated in the definition of $\psi$, and $Z$ is of order $O\left(V_{0}\right)$. It is then possible to show that

$$
G_{s m}=V_{0}\left(\frac{\left\langle\hat{N}_{m} Z\right\rangle_{\mu V T}}{\left\langle\hat{N}_{m}\right\rangle_{\mu V T}\langle Z\rangle_{\mu V T}}-1\right)=V_{0}\left(\frac{\langle Z\rangle_{\mu V T, m}}{\langle Z\rangle_{\mu V T}}-1\right),
$$

where $\langle\cdots\rangle_{\mu V T, m}$ is the grand-canonical ensemble average with a single micellar aggregate whose center is fixed at the origin. ${ }^{32}$ While $\langle\cdots\rangle_{\mu V T}$ is the average for an homogeneous system, $\langle\cdots\rangle_{\mu V T, m}$ is for an inhomogeneous system with an external field produced by a micellar aggregate at the origin. Since the position of the solute is not restricted,

$$
\frac{\langle Z\rangle_{m}}{V_{0}}=\frac{\langle Z\rangle}{V_{0}}+o(1)
$$

holds, where $\langle\cdots\rangle_{m}$ and $\langle\cdots\rangle$ represent the ensemble averages with and without the external field by the micelle, respectively, and $o(1)$ denotes a variable which vanishes in the limit of large system size (thermodynamic limit). In Eq. (A4), the suffix $\mu V T$ is omitted since the equation is valid in any ensemble.

To show the equivalence of Eqs. (10) and (A3), we connect the grand-canonical and isothermal-isobaric ensemble averages with a canonical ensemble average. When the average numbers of particles in the grand-canonical ensemble are adopted as the (input) numbers of particles in the canonical ensemble, $\langle Z\rangle_{\mu V T, m}$ is related to the corresponding canonical ensemble average by

$$
\langle Z\rangle_{\mu V T, m}=\langle Z\rangle_{N V T, m}+\frac{k_{B} T}{2} \sum_{i, j} \frac{\partial N_{i}}{\partial \mu_{j}} \frac{\partial^{2}}{\partial N_{i} \partial N_{i}}\langle Z\rangle+o(1),
$$

where $\langle\cdots\rangle_{N V T, m}$ is the canonical ensemble average with an external field by the micelle at the origin, $N_{i}$ and $\mu_{i}$ are the number and chemical potential of the $i$ th species, respectively. ${ }^{30,75}$ In the second term of Eq. (A5), the ensemble is not necessary to be specified for $\langle Z\rangle$ since the ensemble dependence leads to an $o(1)$ correction. The second term is of order $O(1)$ and represents a nonvanishing effect of the ensemble dependence, where $O(1)$ denotes a variable which does not grow with $V_{0}$ in the thermodynamic limit. The last term is of $o(1)$ due to $Z=O\left(V_{0}\right)$.

An expression similar to Eq. (A5) holds also for $\langle Z\rangle_{\mu V T}$. Actually, the second term of Eq. (A5) deviates from the corresponding term for $\langle Z\rangle_{\mu V T}$ only by $o(1)$ regardless of the presence of the micelle at the origin. The terms beyond the first are thus coincident within $o(1)$ between $\langle Z\rangle_{\mu V T, m}$ and $\langle Z\rangle_{\mu V T}$. It should be noted that the (input) number of particles is different between $\langle\cdots\rangle_{N V T, m}$ and $\langle\cdots\rangle_{N V T}$ by $O(1)$, where $\langle\cdots\rangle_{N V T}$ is the average in the canonical ensemble without the micelle. Since the solute and micelle are dilute, only the difference for water needs to be taken into account as the number of particles for the canonical ensemble. The difference is determined by the Kirkwood-Buff integral $G_{m w}$ between the micelle and water in a form similar to Eq. (A3), and $G_{s m}$ reduces by virtue of Eq. (A4) to

$$
G_{s m}=\frac{V_{0}}{\langle Z\rangle}\left(\langle Z\rangle_{N V T, m}-\langle Z\rangle_{\tilde{N} V T}\right)+\frac{G_{m w} N_{w}}{\langle Z\rangle} \frac{\partial\langle Z\rangle_{N V T}}{\partial N}+o(1),
$$

where $N_{w}$ is the number of water molecules for $\langle\cdots\rangle_{N V T}$, and $\langle\cdots\rangle_{N} V T$ is the average in the canonical ensemble for which a micelle is not involved and the number of particles is equal to that for the ensemble with a micelle up to the order $O(1)$. The ensemble is not necessary to be specified for the average of $Z$ when it is written as $\langle Z\rangle$ in Eq. (A6). In such cases, the ensemble dependence leads only to an $o(1)$ correction.

We now turn to the isothermal-isobaric ensemble averages. According to Eqs. (2) and (4), the difference between $\Delta \mu_{m}$ and $\Delta \tilde{\mu}_{w}$ is expressed as

$$
\exp \left(-\beta\left\{\Delta \mu_{m}-\Delta \tilde{\mu}_{w}\right\}\right)=\frac{\langle V Z\rangle_{P V T, m}\left\langle V^{2}\right\rangle_{P V T}}{\left\langle V^{2}\right\rangle_{P V T, m}\langle V Z\rangle_{P V T}},
$$

where $\langle\cdots\rangle_{P V T, m}$ and $\langle\cdots\rangle_{P V T}$ are the isothermal-isobaric ensemble averages with and without a micellar aggregate fixed at the origin, respectively, and the intramolecular contribution of the solute in the absence of the solute-solvent interaction is considered to be independent of the system volume. The connection of Eq. (A7) to the canonical ensemble counterpart can be seen by noting

$$
\frac{\langle V Z\rangle_{P V T, m}}{\langle V\rangle_{P V T, m}}=\langle Z\rangle_{N V T, m}+\frac{k_{B} T \kappa_{T}}{2} \frac{\partial^{2}}{\partial V_{0}^{2}} V_{0}\langle Z\rangle+o(1),
$$

and the corresponding expressions for $\langle V Z\rangle_{P V T},\left\langle V^{2}\right\rangle_{P V T, m}$, and $\left\langle V^{2}\right\rangle_{P V T}$, where the average volume in the isothermalisobaric ensemble is taken to be the (input) volume of the canonical ensemble and $\kappa_{T}$ is the isothermal compressibility of the system. ${ }^{30,75}$ As in the case of Eq. (A5), the terms beyond the first in the expression of the form of Eq. (A8) are coincident within $o(1)$ between $\langle V Z\rangle_{P V T, m}$ and $\langle V Z\rangle_{P V T}$ and between $\left\langle V^{2}\right\rangle_{P V T, m}$ and $\left\langle V^{2}\right\rangle_{P V T}$. The (input) volume is different by $O(1)$, on the other hand, between $\langle\cdots\rangle_{N V T, m}$ and $\langle\cdots\rangle_{N V T}$. The volume of the system changes by the excess partial molar volume $\Delta V_{m}$ of the micelle upon its insertion at 
the origin. As done for deriving Eq. (A6) from Eq. (A3), we rewrite Eq. (A7) as

$$
\begin{aligned}
V_{0}\left[\exp \left(-\beta\left\{\Delta \mu_{m}-\Delta \tilde{\mu}_{w}\right\}\right)-1\right] \\
=\frac{V_{0}}{\langle Z\rangle}\left(\langle Z\rangle_{N V T, m}-\langle Z\rangle_{N \tilde{V} T}\right) \\
\quad+\frac{\Delta V_{m} V_{0}^{2}}{\langle Z\rangle} \frac{\partial}{\partial V_{0}}\left(\frac{\langle Z\rangle_{N V T}}{V_{0}}\right)+o(1),
\end{aligned}
$$

where $\langle\cdots\rangle_{N \tilde{V} T}$ is the average in the canonical ensemble without a micelle for which the volume is equal to that with a micelle up to $O(1)$.

In the first terms of Eqs. (A6) and (A9), the number of particles and the volume are the same up to $O(1)$ with and without the external field produced by the micelle. The first term is thus coincident within $o(1)$ between Eqs. (A6) and (A9). The second term represents the effect of the overall change in the number or the volume. Actually, $\Delta V_{m}=-G_{m w}$ holds since the solute and micelle are dilute. Furthermore, $\langle Z\rangle / V_{0}$ is an intensive variable, and the second terms of Eqs. (A6) and (A9) become equal in the thermodynamic limit. Equation (10) is therefore proved since $\left(\Delta \mu_{m}-\Delta \tilde{\mu}_{w}\right)$ is of order $O\left(1 / V_{0}\right)$.

When the integrand of Eq. (A2) at a solute configuration $\psi$ is denoted by $z(\psi)$, it is possible to show that

$$
G_{s m}=\frac{V_{0}}{\langle Z\rangle} \int d \psi\left[\langle z(\psi)\rangle_{N V T, m}-\langle z(\psi)\rangle_{N V T}\right]+o(1)
$$

It should be noted in Eq. (A10) that when the (input) volume of the canonical ensemble is $V_{0}$ for $\langle\cdots\rangle_{N V T}$, it is equal to $V_{0}+\Delta V_{m}$ for $\langle\cdots\rangle_{N V T, m}$. An argument similar to that given in Ref. 48 can then be developed and prove that $\langle z(\psi)\rangle_{N V T, m}$ and $\langle z(\psi)\rangle_{N P T, m}$ deviate from $\langle z(\psi)\rangle_{N V T}$ and $\langle z(\psi)\rangle_{N P T}$, respectively, only by $o\left(1 / V_{0}\right)$ terms at the $\psi$ corresponding to the limit of large distance from the micelle. Thus, the thermodynamic limit and the limit of integrating over the whole system can be interchanged in Eq. (A10), and the nonlocal component in the sense introduced in Ref. 48 is absent for $G_{s m}$ when the local estimator is $\langle z(\psi)\rangle$. In the case of $K_{m}$, a similar argument shows that the nonlocal component is present with respect to the local estimator $\langle z(\psi)\rangle$ and that the region far from the micelle makes a nonvanishing contribution in the thermodynamic limit. Actually, we introduced an auxiliary free-energy change $\Delta \tilde{\mu}_{w}$ by Eq. (4) to identify the local component for the micelle-induced change in the solvation free energy of the solute. The extra $V$ factor in Eq. (4) serves to simplify the complications arising from the ensemble dependence and the volume difference in the presence and absence of a micelle.

${ }^{1}$ Handbook of Surface and Colloid Chemistry, 2nd ed., edited by K. S. Birdi (CRC, Boca Raton, FL, 2003).

${ }^{2}$ Solubilization in Surfactant Aggregates, edited by S. D. Christian and J. F. Scamehorn (Marcel Dekker, New York, 1995).

${ }^{3}$ S. H. Yalkowsky, Solubility and Solubilization (Oxford University Press, New York, 1999).

${ }^{4}$ R. Nagarajan and E. Ruckenstein, Langmuir 7, 2934 (1991).

${ }^{5}$ S. Karaborni, N. M. van Os, K. Esselink, and P. A. J. Hilbers, Langmuir 9, 1175 (1993).
${ }^{6}$ P. H. Nelson, T. A. Hatton, and G. C. Rutledge, J. Chem. Phys. 110, 9673 (1999).

${ }^{7}$ B. Jönsson, O. Edholm, and O. Teleman, J. Chem. Phys. 85, 2259 (1986).

${ }^{8}$ K. Watanabe, M. Ferrario, and M. L. Klein, J. Phys. Chem. 92, 819 (1988).

${ }^{9}$ K. Watanabe and M. L. Klein, J. Phys. Chem. 93, 6897 (1989).

${ }^{10}$ J. J. Wendoloski, S. J. Kimatian, C. E. Schutt, and F. R. Salemme, Science 243, 636 (1989).

${ }^{11}$ J. Shelley, K. Watanabe, and M. L. Klein, Int. J. Quantum Chem., Quantum Biol. Symp. 17, 103 (1990).

${ }^{12}$ A. D. MacKerell, Jr., J. Phys. Chem. 99, 1846 (1995).

${ }^{13}$ H. Kuhn and H. Rehage, Ber. Bunsenges. Phys. Chem. 101, 1485 (1997).

${ }^{14}$ H. Kuhn and H. Rehage, Ber. Bunsenges. Phys. Chem. 101, 1493 (1997).

${ }^{15}$ S. Bogusz, R. M. Venable, and R. W. Pastor, J. Phys. Chem. B 104, 5462 (2000).

${ }^{16}$ D. P. Tieleman, D. van der Spoel, and H. J. C. Berendsen, J. Phys. Chem. B 104, 6380 (2000).

${ }^{17}$ S. J. Marrink, D. P. Tieleman, and A. E. Mark, J. Phys. Chem. B 104, 12165 (2000).

${ }^{18}$ J. C. Shelley and M. Y. Shelley, Curr. Opin. Colloid Interface Sci. 5, 101 (2000).

${ }^{19}$ S. Bogusz, R. M. Venable, and R. W. Pastor, J. Phys. Chem. B 105, 8312 (2001).

${ }^{20}$ H. Kuhn, B. Breitzke, and H. Rehage, J. Colloid Interface Sci. 249, 152 (2002).

${ }^{21}$ C. D. Bruce, M. L. Berkowitz, L. Perera, and M. D. E. Forbes, J. Phys. Chem. B 106, 3788 (2002).

${ }^{22}$ C. D. Bruce, S. Senapati, M. L. Berkowitz, L. Perera, and M. D. E. Forbes, J. Phys. Chem. B 106, 10902 (2002).

${ }^{23}$ S. Garde, L. Yang, J. S. Dordick, and M. E. Paulaitis, Mol. Phys. 100, 2299 (2002).

${ }^{24}$ P. J. Bond, J. M. Cuthbertson, S. S. Deol, and M. S. P. Sansom, J. Am. Chem. Soc. 126, 15948 (2004).

${ }^{25}$ A. F. de Moura and L. C. G. Freitas, Chem. Phys. Lett. 411, 474 (2005).

${ }^{26}$ N. Matubayasi and M. Nakahara, J. Chem. Phys. 113, 6070 (2000).

${ }^{27}$ N. Matubayasi and M. Nakahara, J. Chem. Phys. 117, 3605 (2002); 118, 2446 (2003)

${ }^{28}$ N. Matubayasi and M. Nakahara, J. Chem. Phys. 119, 9686 (2003).

${ }^{29}$ J. P. Hansen and I. R. McDonald, Theory of Simple Liquids (Academic, London, 1986).

${ }^{30}$ M. P. Allen and D. J. Tildesley, Computer Simulation of Liquids (Oxford University Press, Oxford, 1987).

${ }^{31}$ The drawback of the approach is, of course, that the solvation free energy is evaluated from an approximate functional.

${ }^{32}$ A. Ben-Naim, Solvation Thermodynamics (Plenum, New York, 1987).

${ }^{33}$ Actually, the requirement of the isobaric correspondence between the micellar system and neat solvent has a deeper meaning, as described in Ref. 49 within the context of the spatial decomposition of Sec. II B.

${ }^{34}$ Actually, the critical micelle concentration cannot be sharply defined and depends on the method of its determination. This is because a micellar aggregate is finite in size and the transition of formation is not sharp. Due to the diffuse nature of the transition, the counterion binding to a micelle formed by ionic surfactants may be significant even at a surfactant concentration "just above" the critical micelle concentration. When the surfactant is ionic, the micelle is a polyion and a high dilution is necessary to neglect the interaction with the counterions. The critical micelle concentration may then not be "dilute enough" for the micelle-counterion interaction in some methods of its determimation; the counterion binding is often a matter of debate in surfactant systems. On the other hand, the distinct aggregates are well separated and their interactions can be safely neglected when the surfactant concentration is close to the critical micelle concentration.

${ }^{35}$ It should be noted that the one-to-one association is implied in Eq. (2). This is because the micelle and solute are both dilute in the present formulation. To treat more than one solute molecule in a micelle, it suffices to extend the development straightforwardly by viewing one of the solute molecules as the "solute" species and the others as the "solvent" species constituting the mixed solvent system.

${ }^{36}$ Of course, the extension of the formulation is straightforward when the system contains cosolvents.

${ }^{37}$ A single solute molecule is contained in Eqs. (3) and (4). In this case, since the average volume of the system is different by $O(1)$ with and 
without the solute, the volume change upon insertion of the solute affects only the $o\left(1 / V_{0}\right)$ term in Eq. (5).

${ }^{38}$ The condition of a fixed (total) chemical potential of the solute is realized, for example, when the counterpart of the equilibrium of the solute insertion is common to the micellar solution and neat solvent.

${ }^{39}$ In principle, $\left(K_{m}+V_{m}\right)$ can have any sign. On the other hand, the expression relating $K_{m}$ to the micelle-water partition coefficient in the mole fraction scale is valid only when $\left(K_{m}+V_{m}\right)$ is positive.

${ }^{40}$ Typically, the standard free energy $\Delta G$ of transfer from bulk water to the micellar pseudophase is introduced with respect to the partition coefficient $K_{x}$ in the mole fraction scale through $\Delta G=-k_{B} T \log K_{x}$ (Refs. 1-3). When $S(0)\left(K_{m}+V_{m}\right) \gg n_{A}$, however, $K_{x}$ is simply close to $C_{w} / S(0)$ and is determined essentially by the solubility in the neat solvent. Indeed, when the affinity of the solute to the micelle is strong enough, the mole fraction in the micellar pseudophase is at the order of 1 and the order of $K_{x}$ is given by the solubility in neat water for a sparingly soluble solute. Therefore, the comparison between the experimental $\Delta G$ and the computational $\Delta G$ constructed from $K_{m}$ is not quantitatively informative when $S(0)$ $\times\left(K_{m}+V_{m}\right) \gg n_{A}$ holds. Our calculated values of $K_{m}$ for methane, benzene, and ethylbenzene in sodium dodecyl sulfate micelle satisfy $S(0)$ $\times\left(K_{m}+V_{m}\right) \gg n_{A}$ for the experimental $S(0)$ when $V_{m}$ is considered to be positive.

${ }^{41}$ J. G. Kirkwood and F. P. Buff, J. Chem. Phys. 19, 774 (1951).

${ }^{42}$ I. L. Shulgin and E. Ruckenstein, J. Chem. Phys. 123, 054909 (2005).

${ }^{43}$ In Eq. (8), the micellar aggregate is treated as a single particle. The configuration fluctuates within the aggregate, and the coordinates for the intra-aggregate motion are incorporated in the definition of $\mathbf{x}$.

${ }^{44}$ Since a single micelle is contained in Eq. (2), $\left(\Delta \mu_{m}-\Delta \mu_{w}\right)$ and $\left(\Delta \mu_{m}\right.$ $\left.-\Delta \tilde{\mu}_{w}\right)$ are of order $O\left(1 / V_{0}\right) . K_{m}$ of Eq. (9) and $G_{s m}$ of Eq. (10) are then of order $O(1)$. Furthermore, since the average volume is different by $O(1)$ between the micellar solution and neat solvent systems, $V_{0}$ in Eqs. (9) and (10) can refer to either of the system volumes. A similar argument applies to the effect of solute insertion, and it does not matter in the limit of large system size whether $V_{0}$ denotes the volume before the solute insertion or the one after. Of course, the remark holds equally in the discussion of Eqs. (16) and (A1).

${ }^{45}$ When the region corresponding to $\Theta_{h}$ is finite in size, $p_{h}$ is of order $O\left(1 / V_{0}\right)$ and $\left(\Delta \mu_{m h}-\Delta \tilde{\mu}_{w}\right)$ is of order $O(1)$. When the region in question is far from the micelle, the corresponding $p_{h}$ is of order $O(1)$ and $\left(\Delta \mu_{m h}-\Delta \tilde{\mu}_{w}\right)$ is of order $O\left(1 / V_{0}\right)$. Thus, $p_{h}\left[\exp \left(-\beta\left(\Delta \mu_{m h}-\Delta \tilde{\mu}_{w}\right)\right)-1\right]$ is of order $O\left(1 / V_{0}\right)$ for each $h$, and Eq. (15) follows from Eqs. (10) and (13) when the $o(1)$ terms are neglected. Equation (15) is exact in the sense that the $o(1)$ terms are not counted (thermodynamic limit).

${ }^{46}$ Since one of $p_{h}$ and $\left[\exp \left(-\beta\left(\Delta \mu_{m h}-\Delta \tilde{\mu}_{w}\right)\right)-1\right]$ is of order $O\left(1 / V_{0}\right)$ and the other is $O(1)$, a remark similar to that given in Ref. 44 holds for $V_{0}$ and the value of $v_{h}$ does not depend on the choice of $V_{0}$ in the limit of large system size $\left(V_{0} \rightarrow \infty\right)$.

${ }^{47}$ The replacement of $\Delta \widetilde{\mu}_{w}$ with $\Delta \mu_{w}$ is not an approximate procedure in the thermodynamic limit when the $h$ th region concerned involves a finite volume. When the volume in question becomes infinite in the thermodynamic limit, the replacement is not allowed as an exact procedure. The replacement of $\Delta \tilde{\mu}_{w}$ with $\Delta \mu_{w}$ for all $h$ in the sum of Eq. (15) is an approximate procedure and corresponds to $G_{s m} \approx K_{m}$

${ }^{48}$ N. Matubayasi, E. Gallicchio, and R. M. Levy, J. Chem. Phys. 109, 4864 (1998).

${ }^{49}$ When the micellar solution and neat solvent are compared at the same volume and $\Theta_{h}$ specifies the region corresponding to the limit of large distance from the micelle, the difference between $\Delta \mu_{m h}$ and $\Delta \tilde{\mu}_{w}$ is of order $O\left(1 / V_{0}\right)$, where $V_{0}$ is the (average) volume of the system (Ref. 48). In this case, the region is of order $O\left(V_{0}\right)$ in size and makes a nonvanishing contribution to the Kirkwood-Buff integral $G_{s m}$ in the thermodynamic limit, as shown generally in Ref. 48. Thus, the isochoric treatment of the solubilization is unfavorable due to the persistence of the nonlocal component of $G_{s m}$ in the sense given in Ref. 48. When the comparison between the micellar solution and neat solvent is made at the same pressure, on the other hand, the difference between $\Delta \mu_{m h}$ and $\Delta \tilde{\mu}_{w}$ is of order $o\left(1 / V_{0}\right)$ for the region with $\Theta_{h}$ corresponding to the limit of large distance from the micelle. The contribution from the region to $G_{s m}$ can then be neglected. The isobaric treatment is therefore desirable within the argument concerning the nonlocal component described in Ref. 48.

${ }^{50}$ N. Matubayasi, L. H. Reed, and R. M. Levy, J. Phys. Chem. 98, 10640 (1994).

${ }^{51}$ N. Matubayasi and R. M. Levy, J. Phys. Chem. 100, 2681 (1996).

${ }^{52}$ When the mixed solvent consists of $n$ components and the energy coordinate for the $k$ th solvent species is $\epsilon_{k}(k=1, \ldots, n)$, the relevant solutesolvent interaction potential is an array of functions given by $\left(u_{1}\left(\epsilon_{1}\right), \ldots, u_{n}\left(\epsilon_{n}\right)\right)$. The corresponding distribution function is then expressed in array form as $\left(\left\langle\hat{\rho}_{1}^{e}\left(\epsilon_{1}\right)\right\rangle_{u}, \ldots,\left\langle\hat{\rho}_{n}^{e}\left(\epsilon_{n}\right)\right\rangle_{u}\right)$ where the average $\langle\cdots\rangle_{u}$ is taken under the solute-solvent interaction $\left(u_{1}\left(\epsilon_{1}\right), \ldots, u_{n}\left(\epsilon_{n}\right)\right)$. The oneto-one correspondence between the set of potential functions $\left(u_{1}\left(\epsilon_{1}\right), \ldots, u_{n}\left(\epsilon_{n}\right)\right)$ defined over the energy coordinates $\epsilon_{k}(k=1, \ldots, n)$ and the set of distribution functions $\left(\left\langle\hat{\rho}_{1}^{e}\left(\epsilon_{1}\right)\right\rangle_{u}, \ldots,\left\langle\hat{\rho}_{n}^{e}\left(\epsilon_{n}\right)\right\rangle_{u}\right)$ can be proved through a procedure similar to that given in Refs. 26 and 28 when $u_{k}\left(\epsilon_{k}\right)=0$ at $\epsilon_{k}=0$ is satisfied for each $k=1, \ldots, n$.

${ }^{53}$ See Appendix B of Ref. 28 for the detailed scheme to determine $w_{k, 0}^{e}$. The procedure for setting the potential zero is applied to each $k$.

${ }^{54}$ K. J. Schweighofer, U. Essmann, and M. Berkowitz, J. Phys. Chem. B 101, 3793 (1997).

${ }^{55}$ J.-P. Ryckaert and A. Bellemans, Chem. Phys. Lett. 30, 123 (1975).

${ }^{56}$ S. J. Weiner, P. A. Kollman, D. A. Case, U. C. Singh, C. Ghio, G. Alagona, S. Profeta, Jr., and P. Weiner, J. Am. Chem. Soc. 106, 765 (1984).

${ }^{57}$ H. Dominguez and M. L. Berkowitz, J. Phys. Chem. B 104, 5302 (2000).

${ }^{58}$ W. L. Jorgensen, J. D. Madura, and C. J. Swenson, J. Am. Chem. Soc. 106, 6638 (1984)

${ }^{59}$ S. J. Weiner, P. A. Kollman, D. T. Nguyen, and D. A. Case, J. Comput. Chem. 7, 230 (1986).

${ }^{60}$ W. L. Jorgensen, J. Chandrasekhar, J. D. Madura, R. W. Impey, and M. L. Klein, J. Chem. Phys. 79, 926 (1983).

${ }^{61}$ W. L. Jorgensen and D. L. Severance, J. Am. Chem. Soc. 112, 4768 (1990).

${ }^{62}$ W. L. Jorgensen, D. S. Maxwell, and J. Tirado-Rives, J. Am. Chem. Soc. 118, 11225 (1996).

${ }^{63}$ W. D. Cornell, P. Cieplak, C. I. Bayly, I. R. Gould, K. M. Merz, Jr., D. M. Ferguson, D. C. Spellmeyer, T. Fox, J. W. Caldwell, and P. A. Kollman, J. Am. Chem. Soc. 117, 5179 (1995).

${ }^{64}$ J. M. Chen, T. M. Su, and C. Y. Mou, J. Phys. Chem. 90, 2418 (1986).

${ }^{65}$ G. J. Martyna, D. J. Tobias, and M. L. Klein, J. Chem. Phys. 101, 4177 (1994).

${ }^{66}$ G. J. Martyna, M. L. Klein, and M. Tuckerman, J. Chem. Phys. 97, 2635 (1992).

${ }^{67}$ W. C. Swope, H. C. Andersen, P. H. Berens, and K. R. Wilson, J. Chem. Phys. 76, 637 (1982)

${ }^{68}$ N. Matubayasi and M. Nakahara, J. Chem. Phys. 110, 3291 (1999).

${ }^{69}$ H. C. Andersen, J. Comput. Phys. 52, 24 (1983).

${ }^{70}$ For all the solutes and regions of the solute position, $\rho_{k, 0}^{e}$ and $\chi_{k l, 0}^{e}$ can be constructed from a single trajectory of the micellar system without the solute since the solute insertion as a test particle does not affect the trajectory. This is useful to reduce the computational demand when a multiple set of solvation is to be treated against a common (mixed) solvent system. A similar remark applies to the calculations in neat water.

${ }^{71}$ Of course, the division of the micellar system is not unique. The boundaries of the regions cannot be sharply defined. The division is introduced only for conceptual convenience.

${ }^{72}$ Of course, $P_{h}$ is not exactly equal to the probability. For instance, the probability is non-negative while $P_{h}$ can be negative. $P_{h}$ and the probability are hardly distinguishable only within the ordinate scale of Fig. 5.

${ }^{73}$ R. E. Wasylishen, J. C. T. Kwak, Z. Gao, E. Verpoorte, J. B. MacDonald, and R. M. Dickson, Can. J. Chem. 69, 822 (1991).

${ }^{74}$ B. E. Hawrylak and D. G. Marangoni, Can. J. Chem. 77, 1241 (1999).

${ }^{75}$ J. L. Lebowitz, J. K. Percus, and L. Verlet, Phys. Rev. 153, 250 (1967). 